\title{
Study of the Effect of Different Sour Stimulus Concentrations and Circadian Rhythm on Salivary Volume by the Modified Ion Dilution Method
}

\author{
Hiroya Gotouda, ${ }^{1,5}$ Hirofumi Sasai, ${ }^{2,5}$ Chieko Taguchi, ${ }^{1}$ Jing Wang, ${ }^{1}$ Kazumune Arikawa, ${ }^{1,5}$ \\ Kayo Kuyama, ${ }^{3,5}$ Junichi Mega,, ${ }^{4,5}$ Hirotsugu Yamamoto,,5 and Seigo Kobayashi, ${ }^{1,5}$
}

Departments of ${ }^{1}$ Community Oral Health, ${ }^{2}$ Public Health and Policy, ${ }^{3}$ Oral Pathology, ${ }^{4}$ Dentistry for the Disabled, ${ }^{5}$ Research Institute of Oral Science, Nihon University School of Dentistry at Matsudo, Matsudo, Chiba 271-8587, Japan

Correspondence to :

Hiroya Gotouda

E-mail : hiroya@mascat.nihon-u.ac.

jp

Keywords :

salivary volume, circadian rhythm, modified fluoride dilution method, sour stimulation

\begin{abstract}
In the current study, we used the modified ion dilution method to evaluate the influence of the circadian rhythm on the salivary volume in response to varying sour concentrations. We found that the salivary volume changed depending on when the sample was taken and on the sour concentration. Specifically, there was a definite trend for the salivary volume to be low in the morning and to increase from around lunchtime to evening. In addition, the influence of the circadian rhythm on salivary volume tended to be reduced by a strong taste stimulation (high sour concentration). These results suggest that the greater the amount of stimulation (sour stimulation), the smaller the effect of circadian rhythm on the salivary volume.
\end{abstract}

\section{Introduction}

Masticatory and taste stimulation methods are used to measure whole salivary volume during stimulation (1). In the taste stimulation method, continuous sour stimulation of the tongue is widely used. However, this method has disadvantages such as a complicated saliva collection procedure and placement of an undue burden on the subjects. Based on the method of Weatherell et al. (2) for measuring the salivary volume, we developed a modified ion dilution method (modified fluoride dilution method [MFD]) and demonstrated its effectiveness in a simplified salivary volume measurement method using sour stimulation (3).

Dawes (4) previously reported the importance of circadian rhythm in the salivary volume. Some studies have examined the influence of the circadian rhythm on the salivary volume because it is thought to be an important factor. In then current study, we used the MFD method to evaluate the salivary volume in relation to the circadian rhythm and varying sour concentrations.

\section{Materials and Methods}

Subjects and salivary collection times

Saliva samples were collected from five subjects (average age $=38.0$ years : 3 males and 2 females) at $10: 00,12: 00,14: 00$, and $16: 00$. The present study was approved (EC 02-029) by the Ethics Committee of the School of Dentistry at Matsudo, Nihon University, Japan. The subjects, who were provided with an adequate explanation, freely agreed to participate in the study.

\section{Collection of saliva by the MFD method}

A NaF (Wako Pure Chemical Industries, Osaka, Japan) solution containing 100 ppm fluoride ion was used as a tasteless stimulation solution, and a solution of $100 \mathrm{ppm}$ fluoride containing 2, 10, or $20 \mathrm{mM}$ tartaric acid (Wako Pure Chemical Industries) was used as a sour stimulation according to the method of Weatherell et al. (2). After thorough washing of the mouth with distilled water, $5.0 \mathrm{ml}$ of the stimulation solution was gargled gently under the tongue for 1 min and then expectorated into a paper cup. 
Determination of fluoride ion concentration and calculation of salivary volume

The fluoride ion concentration recovered in each sample was determined by adding $0.2 \mathrm{ml}$ of TISAB III (Orion, Beverly, MA, USA) to $2.0 \mathrm{ml}$ of the recovered saliva sample followed by analysis with a specific fluoride ion-sensitive electrode connected to a model 290A ion analyzer (Orion). The fluoride multiple electrode was calibrated using fluoride ion standard solutions (Orion) containing fluoride ion at 1,10 , and $100 \mathrm{ppm}$. The salivary volume can be calculated according to the method of Weatherell et al. (2) using the following equation:

$$
\mathrm{F} \times \mathrm{V}=\mathrm{f} \times(\mathrm{V}+\mathrm{X})
$$

Where $\mathrm{F}$ is the fluoride ion concentration in the gargle solution, $\mathrm{V}$ is the volume, $\mathrm{f}$ is the fluoride ion concentration in the collected saliva, and $\mathrm{X}$ is the salivary volume per min (including the salivary volume remaining in the oral cavity immediately before mouthwash). Thus, the salivary volume per min can be calculated from the following equation:

$$
\mathrm{X}=[(\mathrm{F} \times \mathrm{V}) / \mathrm{f}]-\mathrm{V}
$$

\section{Statistical analysis}

Reproducibility was determined by coefficients of variation.

\section{Results}

Saliva was collected from five subjects at $10: 00$, $12: 00,14: 00$, and $16: 00$ with tasteless stimulation and at sour concentrations of 2, 10, and $20 \mathrm{mM}$. Fig. 1 shows the mean salivary volume with tasteless stimulation as well as the mean tartaric acidstimulated salivary volume. The salivary volumes were low in the morning and increased thereafter in all four solutions.

Table 1 indicates the mean salivary volume at the four collections times and the coefficients of variation at different sour concentrations in the five subjects. Except for subject B, the coefficients of variation decreased with increasing tartaric acid concentration. Fig. 2 shows a comparison of the mean co-

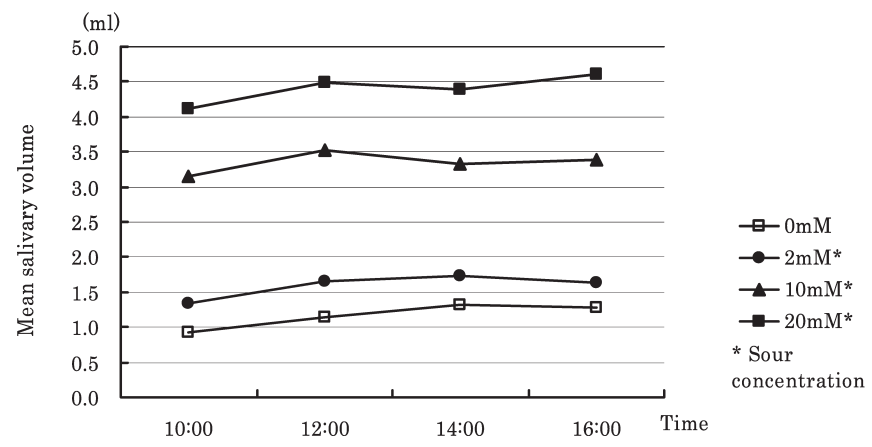

Fig. 1. Changes in the mean salivary volume at different collection times and various sour stimulus concentrations in five subjects

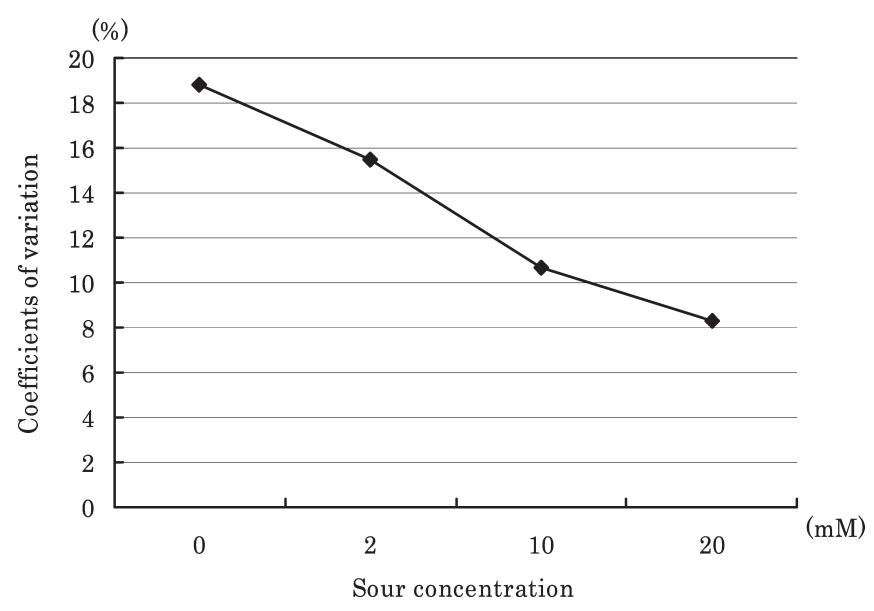

Fig. 2. Total mean coefficient of variation of salivary volume at different sour stimulus concentrations in five subjects

Table 1. Mean salivary volume at the four collection times and coefficients of variation at different sour concentra-

\begin{tabular}{|c|c|c|c|c|c|c|c|c|}
\hline \multirow[b]{2}{*}{ subject } & \multicolumn{2}{|l|}{$0 \mathrm{mM}$} & \multicolumn{2}{|l|}{$2 \mathrm{mM}$} & \multicolumn{2}{|l|}{$10 \mathrm{mM}$} & \multicolumn{2}{|l|}{$20 \mathrm{mM}$} \\
\hline & Mean \pm SD $(\mathrm{ml})$ & $\mathrm{CV}(\%)$ & Mean \pm SD $(\mathrm{ml})$ & $\mathrm{CV}(\%)$ & Mean \pm SD (ml) & $\mathrm{CV}(\%)$ & Mean \pm SD (ml) & CV (\%) \\
\hline $\mathrm{A}$ & $1.03 \pm 0.22$ & 21.3 & $1.36 \pm 0.24$ & 17.6 & $2.90 \pm 0.36$ & 12.5 & $3.72 \pm 0.46$ & 12.3 \\
\hline $\mathrm{B}$ & $1.33 \pm 0.29$ & 21.6 & $1.44 \pm 0.26$ & 18.3 & $2.71 \pm 0.32$ & 11.9 & $3.62 \pm 0.60$ & 16.6 \\
\hline $\mathrm{C}$ & $0.63 \pm 0.10$ & 16.5 & $1.06 \pm 0.15$ & 14.4 & $2.40 \pm 0.32$ & 13.4 & $3.06 \pm 0.14$ & 4.5 \\
\hline $\mathrm{D}$ & $1.16 \pm 0.18$ & 16.0 & $1.57 \pm 0.27$ & 17.0 & $3.19 \pm 0.38$ & 11.8 & $4.09 \pm 0.25$ & 6.1 \\
\hline $\mathrm{E}$ & $1.67 \pm 0.31$ & 18.9 & $2.53 \pm 0.26$ & 10.1 & $5.54 \pm 0.20$ & 3.6 & $7.52 \pm 0.16$ & 2.1 \\
\hline
\end{tabular}
tions in five subjects 
efficients of variation of the salivary volume at different sour concentrations. The coefficient of variation decreased (i.e., the reproducibility increased) as the tartaric acid concentration increased.

\section{Discussion}

Salivary stimulation methods can be classified into two main categories: masticatory and taste stimulation methods $(1,5)$. Watanabe et al. (6) reported that taste stimulation has a greater effect on salivary volume than mechanical stimulation by mastication. Kawamura et al. (7) examined the amount of salivary volume from the human parotid glands using solutions of sucrose, salt, tartaric sour, and quinine hydrochloride as stimulators. They found that the salivary volume decreased in the order of tartaric acid (sourness), salt (saltiness), quinine hydrochloride (bitterness), and sucrose (sweetness). Yamamoto et al. (8) reported that when decerebrated rabbits were given these solutions, the volume from the submandibular glands was the greatest with the tartaric acid solution, followed by salt, quinine hydrochloride, and sucrose solutions. For this reason, we used tartaric acid in the current study to stimulate salivation.

Chauncey et al. (9) stimulated the tongue with acid -soaked cotton and examined the relationship between the salivary volume of the parotid glands and the frequency of stimulation. However, this method had the disadvantages of a complicated salivary collection procedure and an undue burden on the subjects. The MFD method overcomes these disadvantages and makes measurement possible with a simple mouthwash technique. By changing the volume of the mouthwash solution from $1 \mathrm{ml}$, as in the method of Weatherell et al. (2), to $5 \mathrm{ml}$, we believe that the area of taste stimulation on the tongue increased, thereby enhancing the effectiveness of the MFD method.

The role of the circadian rhythm in the salivary volume has been previously studied $(10,11)$. Most results indicate that the maximum volume is reached at noon or in the afternoon, indicating a type of circadian rhythm. Dawes (4) reported that the human salivary volume is most stable between approximate- ly 2: 00 and 4:00 PM. Saliva collection is often done during this time period in many studies, and it is believed that the effect of circadian rhythm can be reduced by standardizing the collection time.

To enable to the use of the MFD method for public health and epidemiological studies with a large number of subjects, we examined the salivary volume in five adult subjects at four times and varying sour concentrations. We found that the salivary volume changed with the circadian rhythm and sour concentration. Specifically, there was a definite trend for the volume to be low in the morning and to increase from around lunchtime to evening. In addition, strong taste stimulation (high sour concentration) tended to decrease the influence of the circadian rhythm on the salivary volume. We believe that the greater the amount of stimulation, the smaller effect of circadian rhythm on the salivary volume.

\section{Acknowledgments}

A part of this study was supported by a grant from the Ministry of Education, Culture, Sports, Science and Technology to promote the 2001 Multidisciplinary Research Project (in 2001-2005).

\section{References}

1. Dowen B, Ulf H: Salivary secretion rate, buffer capacity, and $\mathrm{pH}$. In: Tenovuo JO, editor. Human saliva clinical chemistry and microbiology, Volume I, CRC Press, Florida, 1989. p.25-73.

2. Weatherell JA, Strong M, Robinson C, et al. : Determination of volumes of fluid in the mouth by fluoride dilution. Caries Res, 26 : 94-97, 1992.

3. Gotouda H : Study on methods to assess caries activity-volume of saliva, salivary buffer capacity, and cariogenic bacteria level in caries-susceptible group and caries-free group. Nihon Univ J Oral Sci, 29: 194 $-206,2003$.

4. Dawes C: Circadian rhythms in human salivary flow rate and composition. J Physiol, 220 : 529-545, 1972.

5. Hirasawa M: Studies on salivary flow rates in children-response to taste stimulation and bite force of primary teeth. Jpn J Ped Dent, 38 : 93-103, 2000.

6. Watanabe $\mathrm{S}$ and Dawes $\mathrm{C}$ : The effect of different foods and concentrations of citric acid on the flow rate of whole saliva in man. Arch Oral Biol, 33 : 1-5, 1988.

7. Kawamura Y, Funakoshi M, Nishiyama T, Majima T, Kamata A: Relations between taste qualities and 
parotid gland secretion rate. J Physiol Soc Japan, 26 : 495-502, 1946.

8. Yamamoto T, Kawamura Y: Gustatory-salivary reflex in the rabbit food intake and chemical senses, 211-221, University of Tokyo Press, Tokyo, 1977.

9. Chauncey HH, Feller R, Pshannon IL: Measurement of human gustatory perception using the parotid gland secretion rate, In: Taste and Olfaction II, Oxford Pergamon Press, 1967. p.265-280.
10. Ferguson DB and Botchway CA : Circadian variation in the flow rate and composition of whole saliva stimulated by mastication. Arch Oral Biol, 24: 877881, 1980.

11. Dawes $C$ : Factors influencing salivary flow rate and composition. In: Edgar VM and O'Mullane editors. Saliva and Oral Health, $2^{\text {nd }}$ ed, British Dental Association, London, 1996. p.27-41. 